\title{
Blockchain-based platforms for genomic data sharing: a de-centralized approach in response to the governance problems?
}

\author{
Mahsa Shabani \\ Center for Biomedical Ethics and Law, Department of Public Health and Primary Care, University of Leuven, Leuven, Belgium \\ Corresponding Author: Mahsa Shabani, LLM, PhD, Center for Biomedical Ethics and Law, Department of Public Health \\ and Primary Care, University of Leuven, Kapucijnenvoer 35 B0X 7001, B-3000 Leuven, Belgium (Mahsa.shabani@kuleuven.be)
}

Received 23 August 2018; Revised 12 October 2018; Editorial Decision 16 October 2018; Accepted 18 October 2018

Key words: ethics, policy, data sharing, decentralized, privacy

\begin{abstract}
Blockchain-based platforms are emerging to provide solutions for technical and governance challenges associated with genomic data sharing. Providing capabilities for distributed data stewardship and participatory access control along with effective ways for enforcement of the data access agreements and data ownership are among the major promises of these platforms.
\end{abstract}

\section{BACKGROUND}

Fostering open and responsible genomic data sharing has constituted a core principle of many national and international initiatives such as the All of Us Research Program in the United States and Global Alliance for Genomics and Health. Despite the widespread attention paid to the importance of genomic data sharing, technical and governance bottlenecks hinder data sharing. A recent survey of genomic sequencing initiatives demonstrated that lack of conformity and interoperability of bioinformatics pipelines, lack of financial support, together with legal, consent, and privacy related issues are among the major challenges in front of genomic data sharing. ${ }^{1}$

Some of the challenges associated with the governance of genomic data sharing are rooted in adopting centralized approaches towards storage, sharing, and access to data. The success of centralized data sharing hinges mainly upon functioning and wellresourced central data storage infrastructures and/or centralized data access control services. The previous studies revealed that data custodians are confronting constraints in establishing central data access management mechanisms. ${ }^{2}$ Moreover, the non-automated nature of traditional data sharing and access adds to the complexity of oversight on compliance of data sharing and use with the consent forms and data access agreements. ${ }^{3}$ In addition, the centralized platforms are not suitable in facilitating active participation of multiple stakeholders, such as individuals and patients, in the governance of data sharing.

Distributed networks are suggested as one solution, which enable approved queries to be made to distributed, encrypted databases and allow each independent data contributor to manage the data access. ${ }^{4}$ As such, trust is placed in the network rather than a third party. Distributed networks are perceived to be advantageous, as they aim to overcome the inefficiency, expense, and security risks of transferring datasets to central repositories, often across international boundaries. As Philippakis and colleagues point out, data sharing through such networks, "allows each database to be autonomous with respect to its own data schema, maintain ongoing control of its own data, and continuously innovate at its own pace." 5

One of the emerging examples of distributed networks is the Blockchain-based platform for data sharing and access. Blockchain is a decentralized peer-to-peer architecture with nodes consisting of network participants. Each member in the network stores an identical copy of the Blockchain and contributes to the collective process of validating and certifying digital transactions for the network. Recently, Blockchain-based solutions in the context of genomic- and health-related data have been introduced, with the promise of improving data access, patient empowerment, and improved interoperability. ${ }^{6,7}$ Also in the framework of the "Use of Blockchain in Health IT and Health-Related Research" contest, organized by the Office of the National Coordinator for Information Technology (ONC) in 2016, ${ }^{8}$ use of Blockchain for genomic data sharing by a number of proposals and whitepapers was proposed. 
Table 1. Examples of Blockchain-based platforms and prototypes for genomic and health-related data sharing

\begin{tabular}{|c|c|c|}
\hline Name & Features & Description \\
\hline Encrypgen & $\begin{array}{l}\text { A platform to mediate the searching, storing, buying } \\
\text { and selling of genomic data } \\
\text { Individuals may choose to allow scientists to search } \\
\text { for and buy their data } \\
\text { Putting the individual in charge of his/her genetic } \\
\text { data }\end{array}$ & $\begin{array}{l}\text { "The Gene-Chain is a Blockchain (a cryptographically encoded } \\
\text { ledger) to mediate the searching, storing, buying and selling of } \\
\text { genomic data. It is a private network, meaning that to fully par- } \\
\text { ticipate by searching, one must buy a node through a license, en- } \\
\text { hancing security and privacy of the genetic data on our network. } \\
\text { Anyone is free to safely store their data on the Gene-Chain." } \\
\text { https://encrypgen.com }\end{array}$ \\
\hline Health Nexus & $\begin{array}{l}\text { - Medical data exchange } \\
\text { - Health cash } \\
\text { - Distributed protocol }\end{array}$ & $\begin{array}{l}\text { "Health Nexus is an open-source Blockchain protocol that pro- } \\
\text { vides a more efficient, trustworthy and secure path for the data } \\
\text { to travel. Whether its patients to providers, pharmaceuticals to } \\
\text { researchers, or supply chain to consumers, Health Nexus opens } \\
\text { up a whole marketplace for innovation in data transfer for the } \\
\text { healthcare community." https://tokensale.simplyvitalhealth. } \\
\text { com/ }\end{array}$ \\
\hline Luna Coin & $\begin{array}{l}\text { - Incentivizing patients to share data by offering Luna } \\
\text { Coins }\end{array}$ & $\begin{array}{l}\text { "Luna is a community owned database that rewards individuals } \\
\text { Luna Coins for contributing their DNA and other medical } \\
\text { information." https://www.lunadna.com }\end{array}$ \\
\hline MedRec & $\begin{array}{l}\text { - Patient-vetted data exchange between medical juris- } \\
\text { dictions } \\
\text { - An interoperable content management system } \\
\text { Enabling patients to build a holistic record of their } \\
\text { medical data and authorize others for viewership }\end{array}$ & $\begin{array}{l}\text { "Leveraging unique Blockchain properties, MedRec manages au- } \\
\text { thentication, confidentiality, accountability and data sharing- } \\
\text { crucial considerations when handling sensitive information. A } \\
\text { modular design integrates with providers' existing, local data } \\
\text { storage solutions, facilitating interoperability and making our } \\
\text { system convenient and adaptable. We incentivize medical stake- } \\
\text { holders (researchers, public health authorities, etc.) to partici- } \\
\text { pate in the network as Blockchain 'miners'." https://www. } \\
\text { media.mit.edu/research/groups/1454/medrec }\end{array}$ \\
\hline Nebula Genomics & $\begin{array}{l}\text { - Directly connecting data holders and data buyers } \\
\text { - Sharing data by individuals through the network } \\
\text { while maintaining the ownership of data } \\
\text { - Avoid centralization of databases }\end{array}$ & $\begin{array}{l}\text { "Nebula Genomics will leverage blockchain technology to elimi- } \\
\text { nate the middleman and empower people to own their personal } \\
\text { genomic data. This will effectively lower sequencing costs and } \\
\text { enhance data privacy, resulting in growth of genomic data. Our } \\
\text { open protocol will leverage the genomic data growth by en- } \\
\text { abling data buyers to efficiently aggregate standardized data } \\
\text { from many individuals and genomic databanks." https://www. } \\
\text { nebulagenomics.io }\end{array}$ \\
\hline Opal/Enigma & $\begin{array}{l}\text { - Encourage public-private partnerships } \\
\text { - Ensure data access } \\
\text { - Allow for open data } \\
\text { - Promote "big data" applications in health IT }\end{array}$ & $\begin{array}{l}\text { "An encrypted platform that is able to create a secure environment } \\
\text { for the storage and analysis of healthcare information by using } \\
\text { Blockchain technology, as an effective solution to address the } \\
\text { privacy and security concerns of the stakeholders." http://www. } \\
\text { opalproject.org/about-us/ }\end{array}$ \\
\hline Shivom & $\begin{array}{l}\text { - Genome data donors own their data and access rights } \\
\text { - Donors gain rewards for sharing their genomic data } \\
\text { on the Blockchain } \\
\text { - Pharmaceutical companies use the Shivom platform } \\
\text { to refine drug development }\end{array}$ & $\begin{array}{l}\text { "The objective of the company is to make Shivom the largest geno- } \\
\text { mic data-hub on the planet. Contributors own their genomic } \\
\text { data, the right to access it and the opportunity of rewards for } \\
\text { sharing its information is in the individual's full control."https:// } \\
\text { icos.icobox.io/uploads/whitepaper/2017/11/5a00c78d09c84.pdf }\end{array}$ \\
\hline Zenome.io & $\begin{array}{l}\text { - User uploads the file sequence to Zenome.io platform } \\
\text { - User buys genetic services User sells genetic data }\end{array}$ & $\begin{array}{l}\text { "Zenome is a decentralized market of genomic data and services. } \\
\text { They buy your data. You buy their services. Your personal Ge- } \\
\text { nome Economy." https://zenome.io }\end{array}$ \\
\hline
\end{tabular}

Although still in its infancy, distinctive features of Blockchainmediated technologies can offer novel approaches to address the identified concerns with governance of genomic data sharing. In this paper, I investigate the suitability of proposed Blockchain-based solutions for governance challenges in genomic data sharing, by looking at some of the emerging Blockchain-based platforms and the relevant published whitepapers and proposals (Table 1).

\section{DISTRIBUTED DATA STEWARDSHIP}

A centralized approach to genomic data sharing often entails centralized stewardship for data protection and access. Using such an approach, data are stored in central databases with the access procedure to be controlled by central data access committees. ${ }^{9}$ The database of genotypes and phenotypes (dbGaP) established by NIH is an example of such centralized efforts to streamline data sharing. However, this may constitute a resource-intensive task for a third party, who is responsible to manage data access control for a large number of datasets.

In contrast, Blockchain replaces the centralized infrastructure with a distributed one, proposing a governance mechanism in which authority is distributed among many trusted actors. This will allow the nodes to maintain local stewardship on the databases, "assign a set of access permissions and designate who can query and write 
data to his Blockchain". ${ }^{10}$ Although a similar local stewardship could be provided by traditional distributed networks, Blockchain technology offers further transparency and efficiency in access management, as "access control policies would also be securely stored on a Blockchain and only the [data producer] would be allowed to change them. This provides an environment of transparency and allows [the data producer] to make all decisions about what data is collected and how the data can be shared." ${ }^{11}$ It should be noted that in order to maintain efficiency and scalability of the system, mainly information such as data ownership or viewership permissions is stored on-chain. For instance in the framework of MedRec, "A syncing algorithm handles data exchange 'off-chain' between a patient database and a provider database, after referencing the blockchain to confirm permissions via [their] database authentication server."12

In addition, Blockchain-based platforms provide immutable auditing capabilities, allowing an unchangeable ledger to record critical information across the network. ${ }^{13}$ To date, various methods have been suggested for monitoring data access procedures in the centralized manner, such as institutional audits or requiring the access committees to publish the information related to data access requests and approvals. However, the adequacy of such mechanisms is yet questionable.

\section{PARTICIPATORY ACCESS CONTROL}

One of the main challenges in governance of genomic data sharing is access control. As genomic data may reveal sensitive health- and non-health-related personal information, adopting privacypreserving mechanisms when sharing data is imperative. To date, the access control has been managed in non-automated ways, mainly through access committees who vet the eligibility of data users and allow access to specific datasets or access to the data available on the database. However, the major shortcomings are associated with such controlled access models, including lack of harmonization in access policies, burdensome or bureaucratic access procedures, resource-intensive monitoring, and lack of adequate tools for ongoing oversight.

Blockchain-mediated technologies could transform the current system in a number of ways. First, by adopting a "permissioned" structure of Blockchain, in which only pre-approved users are allowed access to the data, the access control on sensitive genomic data could be managed in an automated way. Although the metadata describing the datasets are available to everyone on the system, that does not mean the data stored within the Blockchain are readable to everyone. Blockchain "relies on pseudoanonymity and public key infrastructure (PKI), which allows the Blockchain's contents to be encrypted in a way that is prohibitively expensive to crack." 14 Implementing such technology could allow discoverability of the existing datasets, while protecting the privacy of the individuals by restricting access to the approved users. In addition, in order to ensure anonymity of the datasets when necessary, Blockchain-based platforms may use tools such as SGX (Software Guard Extension), which leverage multiple cryptographic protocols to enable efficient and secure data storage and computation outsourcing. ${ }^{15}$ In addition, other approaches such as maintaining sensitive data off-chain has been implemented to further protect privacy of sensitive data.

Second, the access control could be managed in a participatory way, as individuals (data subjects), healthcare professionals, and researchers could be part of access control. Currently, the lack of infrastructure and resources is underlined as part of the reason not to include all stakeholders in the management of data access control.
The Blockchain-based solutions can provide the infrastructure in which various stakeholders could be directly involved in the management of data access. As Till and colleagues highlighted: "Blockchain creates the opportunity for medical information to remain the property of the patient, thus allowing an individual to opt in or out of given research studies. This can be accomplished by using the [...] biometric data as a kind of private key, requiring researchers to gain approval and ascent from the patients before using their anonymised health data."16

\section{AUTOMATING ACCESS AGREEMENTS AND CONSENT}

Currently, the terms and conditions of data access such as the purposes of use and co-authorship requirements are incorporated into the data access agreements. Although the access agreements are legally binding documents, there is a lack of tools and mechanisms to effectively monitor the enforcement of the agreements. Consequently, it is difficult to identify the violations of the agreement, and assure the terms and conditions of data access are respected.

In the framework of Blockchain-mediated technologies, access agreements can be integrated into Smart Contracts, which have the capacity to digitally enforce and verify the contract by using computer programs designed to manage smart properties. A well-known example of a smart contract is Ethereum, which intends to provide a Blockchain with a built-in programming language that can be used to create "contracts" that can be used to encode transaction functions. ${ }^{17}$ Individuals may authorize access to their record only under certain conditions or for a specific reason. The advantage of Smart Contract is that it can ensure that patient authorization is codified and executable. In fact, Smart Contracts could automate the process of informed consent. "For example, a patient could create a smart contract that provides anonymized data about his tumor biopsy to any research organization that asks for it. This would happen automatically, without the patient having to explicitly release the information." 14

Second, explicit tracking of academic credits becomes possible by incorporating them into Smart Contracts. One example is when there is an agreement between the data producers and the data users that the data producer should be co-author of the papers resulting from analysis of datasets. In a Blockchain-based platform, it would be possible to put that agreement in a box in the Blockchain on the Internet. "Reviewer, editors, and readers of the paper could then visit the specified Blockchain box on the Internet, read the agreement, and confirm that the authorship conditions were properly fulfilled. The permanent visibility of the agreement makes it impossible for one party to unilaterally repudiate it." 18

In addition, other incentivizing mechanisms in terms of the academic credits have been suggested that could be integrated into Blockchain-based platforms. "Authorship Coin" 18 is one example, which can be used to reward data sharing efforts of the data producers. As the professional concerns of researchers regarding receiving credits when sharing data appears to be a reason for data withholding, ${ }^{19}$ such policies embedded in the Blockchain-based platforms for data sharing are valuable.

\section{DATA OWNERSHIP AND DNA COINS}

Depending on the policies of the genomic test providers, in addition to the report of the results, individuals may get access to their raw 
genomic data. ${ }^{20}$ Despite the legal vacuum regarding proprietary status of genomic data in many jurisdictions, there is an increasing support from scholars, patient groups, and the general public to recognize the individual's ownership rights on raw genomic data and facilitate the access to raw genomic data and related medical records. ${ }^{21}$

The emerging Blockchain-based platforms aim for providing an ecosystem in which individuals could maintain ownership of their personal genomic- and health-related data, and decide how to share their data and under what conditions. Arguably, once individuals are in power to manage their personal data, they will be incentivized to share their genomic data for various purposes. ${ }^{22}$

For instance, some Blockchain-mediated platforms aim for introducing reward systems for personal genomic data sharing. As an example, on the webpage of the Luna Coin, it is indicated that, "Luna rewards people to share the data they already own," as "there is currently little incentive for consumers to contribute their DNA and health information to a third party database." In a similar example, Shivom, proposes that, "patients will be able to monetize their data by anonymously offering controlled access to their health data to pharmaceutical companies. This opens new opportunities to observe wide-reaching patterns in drug development, while still preserving the privacy of patients and lowering the overhead associated with traditional clinical trials. In this way Blockchain technology enables the emergence of new data economics between data producer and data consumer, as the system supplies information to empower researchers while engaging patients and providers in the choice of how much and which metadata to release."23

Although offering rewards for genomic data sharing is not completely a novel strategy, it remains to be seen how introducing monetary values for individuals who share their own genomic data will be managed in various jurisdictions.

\section{IS BLOCKCHAIN A SOLUTION FOR THE PROBLEMS OF GENOMIC DATA SHARING AND BEYOND?}

Blockchain-based platforms target the major challenges in governance of genomic data sharing, among others. The ultimate goal is to ensure that organizations and participants can share data with privacy-preserving algorithms that facilitate compliance to legal and ethical standards. Although the majority of emerging platforms are still in their infancy, they can be considered beneficial in offering novel approaches to address governance challenges in genomic data sharing.

Notably, Blockchain is more than a technological infrastructure-it is a new way of governing open networks, in which the potentials of de-centralized networks, industry need, and consumer genetics are being harvested. Thereby, the main breakthrough here is beyond the technology, but is facilitated by the technology. Blockchain-based networks aiming to scale the amount of data, while providing new models of ownership and facilitating active participation of individuals in the governance of data sharing.

In particular, the Blockchain-based solutions could provide opportunities to automate the procedure of data access control and improve the transparency and fairness in genomic data access. Similarly, enforceability of access agreements could be significantly improved by using Smart Contracts. This is a significant undertaking, given the fact that it will provide reassurance for researchers and data custodians that downstream data uses will be in compliance with the terms and conditions of data uses.

Moreover, if implemented successfully, the Blockchain-based solution may change the culture of data sharing. In particular, the role of patients and individuals in the data-sharing ecosystem could be strengthened, and monopoly of the public and private test providers on management of the genomic data sharing could be diminished. Blockchain has the ability to create new commons that occupy a space between the market and public goods. As the Blcokchainbased platforms aim to enable patients and citizens to control and share their data, a substantial amount of education, design work around incentives, ownership, and participatory control may be required to catalyze this movement.

Yet, the Blockchain-based solutions still need to be backed by legal instruments. Introducing monetary values for individual genomic datasets and ownership rights are the paramount examples of areas in which self-regulation may not be sufficient. Moreover, it is crucial to carefully investigate the potential impacts of such policies in a broader context of biomedical research, and assure that the final policies are in the best interest of science, society, and innovation.

\section{FUNDING}

This work was supported by a postdoctoral fellowship from ResearchFoundation Flanders (FWO). There is no grant number available for this fellowship.

\section{CONTRIBUTORSHIP STATEMENT}

MS is the single contributor of this manuscript.

Conflict of interest statement. The author has no competing interests to declare.

\section{REFERENCES}

1. Vis $\mathrm{D}$, Lewin J, Liao R, et al. Towards a global cancer knowledge network: dissecting the current international cancer genomic sequencing landscape. Ann Oncol 2017; 28 (5): 1145-51.

2. Shabani M, Dyke SO, Joly Y, Borry P. Controlled access under review: improving the governance of genomic data access. PLoS Biol 2015; 13 (12): e1002339.

3. Dyke SO, Philippakis AA, De Argila JR, et al. Consent codes: upholding standard data use conditions. PLoS Genet 2016; 12 (1): e1005772.

4. Popovic JR. Distributed data networks: a blueprint for Big Data sharing and healthcare analytics. Ann N Y Acad Sci 2017; 1387 (1): 105-11.

5. Philippakis AA, Azzariti DR, Beltran S, et al. The Matchmaker Exchange: a platform for rare disease gene discovery. Hum Mutat 2015; 36 (10): 915-21.

6. Kuo T-T, Kim H-E, Ohno-Machado L. Blockchain distributed ledger technologies for biomedical and health care applications. J Am Med Inform Assoc 2017; 24 (6): 1211-20.

7. Mettler M, ed. Blockchain technology in healthcare: the revolution starts here. In: 2016 IEEE 18th International Conference on e-Health Networking, Applications and Services (Healthcom). IEEE; 2016; Munich, Germany.

8. ONC. The "Use of Blockchain in Health IT and Health-Related Research" Ideation Challenge 2016. https://www.healthit.gov/newsroom/ blockchain-challenge. Accessed August 10, 2018.

9. Shabani M, Knoppers BM, Borry P. From the principles of genomic data sharing to the practices of data access committees. EMBO Mol Med 2015; $7(5): 507-9$.

10. Linn LK, Koo MB. Martha Blockchain for Health Data and Its Potential Use in Health IT and Health Care Related Research. 2016. https://www.healthit.gov/sites/default/files/11-74-ablockchainforhealth care.pdf. Accessed November 2, 2018. 
11. IBM. Blockchain: The Chain of Trust and Its Potential to Transform Healthcare - Our Point of View. 2016. https://www.healthit.gov/sites/ default/files/8-31-blockchain-ibm_ideation-challenge_aug8.pdf. Accessed November 2, 2018.

12. Ekblaw A, Azaria A, Halamka J, et al. A Case Study for Blockchain in Healthcare: "MedRec" prototype for electronic health records and medical research data. https://www.healthit.gov/sites/default/files/5-56-onc_ blockchainchallenge_mitwhitepaper.pdf. Accessed November 2, 2018.

13. Angraal S, Krumholz HM, Schulz WL. Blockchain technology: applications in health care. Circ Cardiovasc Qual Outcomes 2017; 10 (9): e003800.

14. Ivan D. Moving Toward a Blockchain-Based Method for the Secure Storage of Patient Records. 2016. https://www.healthit.gov/sites/default/files/ 9-16-drew_ivan_20160804_blockchain_for_healthcare_final.pdf. Accessed November 2, 2018.

15. Chen F, Wang C, Dai W, et al. PRESAGE: privacy-preserving genetic testing via software guard extension. BMC Med Genom 2017; 10 (2): 48.

16. Till BM, Peters AW, Afshar S, Meara JG. From blockchain technology to global health equity: can cryptocurrencies finance universal health coverage? BMJ Glob Health 2017; 2 (4): e000570.
17. A BV. A Next-Generation Smart Contract and Decentralized Application Platform. http://blockchainlab.com/pdf/Ethereum_white_paper-a_next_ generation_smart_contract_and_decentralized_application_platformvitalik-buterin.pdf. Accessed October 10, 2018.

18. intel. Blockchains for Data Sharing in Clinical Research: Trust in a Trustless World. 2016. https://simplecore.intel.com/itpeernetwork/wp-content/ uploads/sites/38/2017/05/Intel_Blockchain_Application_Note1.pdf. Accessed November 2, 2018.

19. Bierer BE, Crosas M, Pierce HH. Data authorship as an incentive to data sharing. Mass Med Soc 2017.

20. Shabani M, Vears D, Borry P. Raw genomic data: storage, access, and sharing. Trends Genet 2018; 34 (1): 8-10.

21. Kish LJ, Topol EJ. Unpatients-why patients should own their medical data. Nat Biotechnol 2015; 33 (9): 921.

22. Roberts JL, Pereira S, McGuire AL. Should you profit from your genome? Nat Biotechnol 2017; 35 (1): 18-20.

23. Shivom. Shivom, the global Genomics-Blockchain Ecosystem. 2016. https://investfeed-prod.s3.amazonaws.com/upload/889acaf0-2cfb-11e8bc3a-2c2283ca7ac8.pdf. Accessed November 2, 2018. 